\title{
Computation of the optimal probability of becoming a cluster head in hierarchical clustered WSNs
}

\author{
Mostafa SAADI \\ MATIC Laboratory \\ Faculty of Sciences \\ Chouaib Doukkali \\ University \\ El Jadida - Morocco
}

\author{
M. Lahcen Hasnaoui \\ Faculty of Sciences \\ Sidi Mohammed Ben \\ Abdellah University \\ Fez, Marocco.
}

\author{
Abderrahim BeniHssane \\ MATIC Laboratory \\ Faculty of Sciences \\ Chouaib Doukkali \\ University \\ El Jadida - Morocco
}

\author{
Mohamed Laghdir \\ MATIC Laboratory \\ Faculty of Sciences \\ Chouaib Doukkali \\ University \\ El Jadida - Morocco
}

\begin{abstract}
Wireless Sensor Networks (WSNs) consist of a large number of energy-limited sensor nodes that are densely deployed in a large geographical region. The main challenge facing us in the design and conception of Wireless Sensor Networks (WSNs) is to find the best way to extend their life span. Therefore, proper energy management technics and communication protocols optimization have received increasing attention. The clustering algorithm is a key technique used to increase the scalability and life span of the network in general. In this paper, to generate clusters of sensors and reduce the cost of communication in them, we used a new approach from stochastic geometry as well as a distributed random algorithm. Moreover, we compute the optimal probability of becoming a cluster head in both cases rectangular and circular area. This study can successfully prolong the network's life span by reducing the total energy dissipation on the network and evenly distributing energy consumption over all sensor nodes. Our results could be used in any form of space deployment of sensors.
\end{abstract}

\section{General Terms:}

Wireless Sensor Networks, Clustering Algorithm

\section{Keywords:}

Energy-Efficient, Poisson Process, Stochastic Geometry, Distributed Random Algorithm

\section{INTRODUCTION}

Numerous are the examples of sensors application's in all types of household appliances such as : washing machines, telephones, vaccum cleaners, $\ldots$ and others devices such as the means of transport, the elevators, and so on. The integration of these sensors in objects which are widely used in our life is booming. It is this ability to "perceive the world" that makes our machines become more autonomous, and many tasks that were previously only possible in direct supervision of the man can be self-organized. A sensor can be defined as a device that receives a physical property which maps value to a quantitative measure. There are, for example simple sensors for position, speed, acceleration, pressure, motion, light, and temperature,...

Besides, there are more complex sensors such as sound or image sensors which are also very widely used. With the integration of sensors in embedded systems, the area of applications has widened. Embedded systems are integrated systems that enable specific functionality to perform very specific tasks (sometimes critical) in complex systems, in addition to their role, for optimized operation in terms of efficiency, reliability and security. If a device needed to communicate with other devices, a cable connection was the only possible solution in a first time. Over time, the cost of silicon chips has continued to decrease exponentially, and in many cases, the cost of radio frequency integrated circuits is now below than those of cable and connectors.

The actual wireless transmission systems add integrated wireless communication without hard wired connections. This integration of sensors, embedded systems and intelligent wireless communication has led to the birth of a new range of electronic devices that pave the way for new applications based on wireless Sensors Networks.

Sensor networking has always been a brain-teasing field of research. A wireless sensor network (WSN) consists of a large number of sensor nodes arbitrarily deployed in an unreachable field to give the end-user the ability to instrument, observe, and react to events and phenomena in a specified environment [4] 1]. WSNs have increasingly been used in so many different fields such as military, health, environment and disaster management. Other applications of these sensors ,to name but a few, include habitat observing, medical fields, smart offices, spotting chemical or nuclear explosive materials and transportation traffic monitoring [1, 15]. Since they are exposed to atrocious and dynamic environments and limited in their energy level, processing power and sensing ability, WSNs must deliver only processed and concise data. Therefore, any inefficient use of these WSNs leads to a poor performance and consequently a short life cycle. Routing techniques are the most important issue for networks where resources are limited. In this respect, there are two types of schemes that operate differently. Conventional centralized algorithms operate with a global knowledge of the whole network and any error in transmission or a failure of a critical node will potentially bring about a serious protocol failure; whereas distributed algorithms are executed locally with partial nodes, which can prevent any failure caused by a single node [7, 8]. The distributed algorithms have proved to be more robust and scalable than centralized ones. when the sensor nodes are organized efficiently into clusters they consume less energy. The clustering techniques are used to design many energy-efficient routing protocols in order to perform data aggregation and increase the WSNs' lifetime. Clustering sensors into groups, so that sensors communicate information only to cluster heads and then the cluster heads communicate the aggregated information to the processing center, save energy [8] 18].

Thus, it is advantageous to organize the sensors into clusters; where the data gathered and fused by the sensors is commu- 
nicated to the BS through a hierarchy of cluster-heads [8]. The cluster-heads, which are elected periodically by certain clustering algorithms, aggregate the data of their cluster members and send it to the base station, from where the end-users can access the sensed data. Thus, only some nodes are required to transmit data over a long distance and the rest of the nodes will need to complete short distance transmission only. Therefore, more energy is saved and the overall network lifetime can be extended. To this end, energy in these sensors is limited and must be managed in an efficient way. In this paper, to generate clusters of sensors and reduce the cost of communication in them, we used a new approach from stochastic geometry as well as a distributed random algorithm. This study can successfully prolong the network's life span by reducing the total energy dissipation on the network and evenly distributing energy consumption over all sensor nodes. Our results could be used in any form of space deployment of sensors.

The remainder of the paper is organized as follows. In Section 2, define the homogeneous poisson process and computation of the optimal probability of becoming a cluster head when the area is a rectangle. Section 2.5 describes the parameters of circular area. Finally, Section 3 gives concluding remarks.

\section{COMPUTATION OF THE OPTIMAL PROBABILITY OF BECOMING A CLUSTER HEAD}

The distributed computation over a network and its application to statistical consensus theory has a long history. To deal with the disadvantages of centralized systems, the distributed algorithms have been an efficient solution and have been a basic tool for achieving globally optimal distributed decisions in a wireless sensor network. In distributed algorithms, sensors interact and communicate with each other, only neighbors, to organize themselves and to reach an agreement regarding a certain value that depends on the state of the sensors. Consensus problems also called agreement problems, have a long history in the field of computer science, particularly in automata theory and distributed computation[14]. This local interaction gives rise to distributed detection or estimation schemes, more accurate than that of each single sensor and capable of achieving globally optimal decisions, without the need to send all the collected data to a fusion center. Moreover, the interaction occurs through a very simple mechanism that does not require complicated modulation, MAC, or routing strategies. This interaction among the sensors is the key feature, as it improves the reliability of the local decisions and, at the same time, it yields fault tolerance and scalability. There is a substantial amount of theoretical works which explain and clarify the importance of this consensus algorithm than a centralized system [12, 17]. The consensus problem introduced by WSNs can be solved using iterative algorithms. The importance of such algorithms which converge on the state is focussed on their robustness to changes in the network topology. Nevertheless, they are sensitive to propagation delays, the noise variance and the energy consumption. Important contributions on consensus algorithms converging on the state can be found in literature [5, 11, 19].

Moreover, when the network size becomes very important, its management becomes more difficult. Using either, the centralized system or the distributed algorithm without fusion center will be complicated.

Consequently, the so called hierarchical clustering can be an intermediate and strong solution for networks composed of a large number of sensors. Hierarchical protocols have been proposed in order to meet the energy efficiency and scalability requirement of the WSNs.

In a WSN, the large part of energy is consumed when the wireless communications are established [10]. With the aim to face this problem, several communication protocols have been pro- posed. In particular, different techniques were proposed to guarantee transmissions providing efficient energies in Ad-hoc networks. Thus, many communication models and protocols that are designed for specific applications, queries, and topologies, exist.

\subsection{Spatial point process}

A spatial point process is a random pattern of points in $d$ dimensional space (where usually $d=2$ or $d=3$ in applications). Spatial point processes are useful as statistical models in the analysis of observed patterns of points, where the points represent the locations of some object of study (trees in a forest, bird nests, disease cases, or petty crimes). Point processes play a special role in stochastic geometry, as the building blocks of more complicated random set models (such as the Boolean model), and as instructive simple examples of random sets.

In probability theory, a Poisson process is a stochastic process which counts the number of events and the time that these events occur in a given time interval.

Poisson point processes play a fundamental role in the theory of point processes. They possess the property of "no interaction" between points or "complete spatial randomness". As such, they are practically useless as a model for a spatial point pattern as most spatial point patterns exhibit some degree of interaction among the points. However, they serve as reference processes when summary statistics are studied and as a building block for more structured point process models.

Spatial Poisson point processes have been used in many branches of science to model various environmental phenomena. These areas include forestry, technology, geodesy, military applications and many more 20, 9].

a. spatial distribution of stars or galaxies;

b. spatial distribution of plants or animals;

c. spatial distribution of bacteria on a slide;

d. spatial distribution of defects on a surface or in a volume;

e. spatial distribution of oil in a region.

\subsection{Definition}

\section{Spatial Poisson Processes (POINT PROCESSES) [2} Notation:

- Let $S$ be a subset of $R^{2}$. ( $\left.R^{k}\right)$ (Assume $\mathrm{S}$ is normalized to have volume 1.)

- Let $\mathfrak{A}$ be the family of all subsets of $S$.

-For $A \epsilon \mathfrak{A}$, let $|A|$ denote the size of $A$. (length, area, volume,...) - Let $N(A)$ be the number of points in the set $A$.

Then $N(A)_{A \in \mathfrak{A} A}$ is a homogenous spatial Poisson process with intensity $\lambda>0$ if:

-For each $A \epsilon \mathfrak{A}, N(A) \sim$ Poisson $(\lambda|A|)$.

-For every finite collection $A_{1}, A_{2}, \ldots, A_{n}$ of disjoint subsets of $S$,

$N(A 1), N(A 2), \ldots, N(A n)$ are independent.

we have:

$$
P(N(A)=n)=\frac{e^{-\lambda|A|}(\lambda|A|)^{n}}{n !} \text { forn }=0,1,2, \ldots
$$

\subsection{Simulation of two-dimensional homogeneous poisson processes}

In this study we concentrate on two simple models. The first model is homogeneous Poisson process on the plane(rectangle, square and circle). The second one is $N$ points independently and uniformly distributed (iud) in a certain region of the plane, including circle and rectangle. There are multiple reasons why these models are important in networking. 
The two-dimensional homogeneous Poisson process (of rate $\lambda_{i}$ 0 ) is defined by the properties that the numbers of points in any finite set of non-overlapping regions having areas in the usual geometric sense are mutually independent, and that the number of points in any region of area $A$ has a Poisson distribution with mean $\lambda \mathrm{A}$ [13]. Note that the number of points in a region $R$ depends on its area, but not on its shape or location. The homogeneous Poisson process arises as a limiting two-dimensional point process with respect to a number of limiting operations.

In considering the two-dimensional homogeneous Poisson process, projection properties of the process depend quite critically on the geometry of the regions considered. These projection properties are simple for rectangular(square) and circular regions $W_{0}$, and make simulation of the homogeneous process quite easy. We consider these two cases separately.

2.3.1 Homogeneous Poisson Processes in a Rectangle: Consider a two-dimensional Homogeneous Poisson Process of rate $\lambda$, so that the number of points in a fixed rectangle $R=\{(x, y): 0 \leq x \leq a, 0 \leq y \leq b\}$ has a Poisson distribution with parameter $\lambda \bar{a} b$.

Assume that a two-dimensional Homogeneous Poisson Process of rate $\lambda$ is observed in a fixed rectangle $R=$ $(x, y): 0 \leq x \leq a, 0 \leq y \leq b$, so that the number of points in $R, N(R)$, has a Poisson distribution with parameter $\lambda a b$. If $N(R)=n>0$ and if $\left(X_{l}, Y_{1}\right),\left(X_{2}, Y_{2}\right), \ldots,\left(X_{n}, Y_{n}\right)$ denote the points, labeled so that $X_{1}<X_{2}<\ldots<X_{n}$, then conditional on having observed $n$ points in $R$, the $X_{1}, X_{2}, \ldots, X_{n}$ are uniform order statistics on $0 \leq x \leq a$, and $Y_{1}, Y_{2}, \ldots ., Y_{n}$ are independent and uniformly distributed on $0 \leq y \leq b$ independent of the $X_{i}$.

We consider that sensors in the wireless sensor network are distributed according to a homogeneous spatial Poisson process of intensity $\lambda$ in 2-dimensional space $W_{0}$. This implies that:

—all $N$ sensors are stochastically independent

- each point is uniformly distributed in $W_{0}$

We assume that:

-All sensors transmit at the same power level and hence have the same radio range $r$.

-A distance of $D$ between any sensor and its cluster head is equivalent to $\lceil D / r\rceil$ hops.

Let $\left(X_{i}, Y_{i}\right)$ denote the position of the sensor $s_{i}$ of the process in 2-dimensional space $W_{0}$ (cf Figure 1).

Given the independent random variables $\mathbf{x}_{i}$ and $\mathbf{y}_{i}$ denote the coordinate of the sensor $s_{i}$,

$$
\begin{aligned}
X_{i} & \sim \mathcal{U}\left(\left[0, x_{0}\right]\right) \\
Y_{i} & \sim \mathcal{U}\left(\left[0, y_{0}\right]\right)
\end{aligned}
$$

with probability densities:

$$
f_{X_{i}}\left(x_{i}\right)= \begin{cases}\frac{1}{a} & \text { for } 0 \leq x \leq a \\ 0 & \text { elsewhere }\end{cases}
$$

and

$$
f_{Y_{i}}\left(y_{i}\right)= \begin{cases}\frac{1}{b} & \text { for } 0 \leq y \leq b \\ 0 & \text { elsewhere }\end{cases}
$$

then

$$
\left(X_{i}, Y_{i}\right) \sim f_{\left(X_{i}, Y_{i}\right)}\left(x_{i}, y_{i}\right)
$$

$X_{i}$ and $Y_{i}$ are independent and uniformly distributed (iud), then:

$$
f_{\left(X_{i}, Y_{i}\right)}\left(x_{i}, y_{i}\right)=f_{X_{i}}\left(x_{i}\right) \times f_{Y_{i}}\left(y_{i}\right)
$$

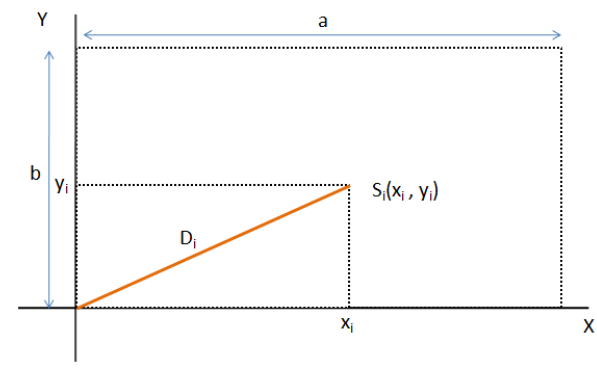

Fig. 1. The distance $D_{i}$

As per our assumptions, the sensors are distributed according to a homogeneous spatial Poisson process and hence, the number of sensors in a fixed rectangle area (cf Figure 1) is a Poisson random variable, $N$, with mean $\lambda A$, where $A=a \times b$.

Let us assume that for a particular realization of the process there are $n$ sensors in this area.

Define $D_{i}$ to be the distance between a sensor located at $\left(x_{i}, y_{i}\right), i=1 ; 2 ; \ldots ; n$ and its own cluster head 1 .

$$
D_{i}=\sqrt{X_{i}^{2}+Y_{i}^{2}}
$$

Then the average distance is:

$$
\begin{aligned}
E\left[D_{i}\right] & =E\left[\sqrt{X_{i}^{2}+Y_{i}^{2}}\right] \\
& =\int_{\mathcal{R}^{2}} \sqrt{x_{i}^{2}+y_{i}^{2}} \cdot f_{\left(X_{i}, Y_{i}\right)}\left(x_{i}, y_{i}\right) d x_{i} d y_{i} \\
& =\int_{\mathcal{R}^{2}} \sqrt{x_{i}^{2}+y_{i}^{2}} \cdot f_{X_{i}}\left(x_{i}\right) \cdot f_{Y_{i}}\left(y_{i}\right) d x_{i} d y_{i} \\
& =\int_{0}^{a} \int_{0}^{b} \sqrt{x_{i}^{2}+y_{i}^{2}} \cdot \frac{1}{a} \cdot \frac{1}{b} d x_{i} d y_{i} \\
& =\frac{1}{a b} \int_{0}^{a} \int_{0}^{b} \sqrt{x_{i}^{2}+y_{i}^{2}} d x_{i} d y_{i} \\
& =\frac{1}{a b} \int_{0}^{a} \int_{0}^{b} \sqrt{x^{2}+y^{2}} d x d y
\end{aligned}
$$

We define $R$ as :

$$
\begin{aligned}
R & =[0, a] \times[0, b] \\
& =\{(x, y): 0 \leq x \leq a, 0 \leq y \leq b\} \\
& =\{(x, y): 0 \leq y \leq x \leq a\} \bigcup\{(x, y): 0 \leq x \leq y \leq b\}
\end{aligned}
$$

then

$$
\begin{aligned}
E\left[D_{i}\right] & =\frac{1}{a b}\left(\int_{0}^{a} d x \int_{0}^{x} \sqrt{x^{2}+y^{2}} d y\right. \\
& \left.+\int_{0}^{b} d y \int_{0}^{y} \sqrt{x^{2}+y^{2}} d x\right)
\end{aligned}
$$

Let $x=y u$ then $d x=y d u$

Let $y=x v$ then $d y=x d v$

then

$$
\begin{aligned}
E\left[D_{i}\right] & =\frac{1}{a b}\left(\int_{0}^{a} d x x^{2} \int_{0}^{1} \sqrt{1+u^{2}} d u\right. \\
& \left.+\int_{0}^{b} d y y^{2} \int_{0}^{1} \sqrt{1+v^{2}} d v\right) \\
& =\frac{1}{a b}\left(\frac{1}{3} a^{3}+\frac{1}{3} b^{3}\right) \int_{0}^{1} \sqrt{1+t^{2}} d t
\end{aligned}
$$




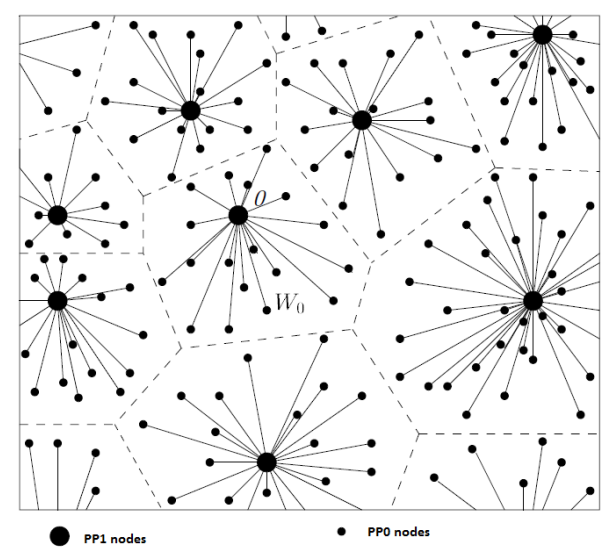

Fig. 2. Voronoi cells

Let:

$$
\int_{0}^{1} \sqrt{1+u^{2}} d u=1,1478
$$

finally we have:

$$
E\left[D_{i}\right]=0,3826 \frac{a^{3}+b^{3}}{a b}
$$

The probability of a sensor becoming a cluster head is $p$; hence, on average, $n p$ sensors will become cluster heads.

Let $D$ be a random variable that denotes the total length of the segments from these cluster heads to the base station. The sensors are distributed according to a homogeneous spatial Poisson process. Hence, given $N$ sensors of the Poisson process in $W_{0}$, these sensors are conditionally iud in $W_{0}$ area. Therefore,

$$
D=\sum_{i=1}^{n p} D_{i}
$$

There are $n$ sensors uniformly distributed in the rectangle area,

$$
\begin{aligned}
E[D \mid N=n] & =\sum_{i=1}^{n p} E\left[D_{i}\right] \\
& =n p E\left[D_{i}\right] \\
& =0,3826 \frac{a^{3}+b^{3}}{a b} n p
\end{aligned}
$$

Now, since a sensor becomes a cluster head with probability $p$, the cluster heads and the non-cluster heads are distributed as per independent homogeneous spatial Poisson processes $P P 1$ and $P P 0$ of intensity $\lambda_{1}=p \lambda$ and $\lambda_{0}=(1-p) \lambda$ respectively.

Each non-cluster head joins the cluster of the closest cluster head to form a Voronoi tessellation[16]. The plane is thus divided into zones called the Voronoi cells, each cell corresponding to a $P P 1$ process point, called its nucleus. If $N_{v}$ is the random variable denoting the number of PP0 process points in each Voronoi cell and $L_{v}$ is the total length of all segments connecting the $P P 0$ process points to the nucleus in a Voronoi cell $W_{0}$, then according to results in [6], and for large values of $n p$,

Let us named:

$-N_{v}$ is the number of PP0 node in the aggregate $W_{0}$.

$-L_{v}$ is the total length of all the segments connecting the PP0 normal nodes of the aggregate $W_{0}$ to its cluster head. then

$$
\begin{aligned}
N_{v} & \sim P P H\left(\lambda_{0}\right) \\
L_{v} & \sim P P H\left(\lambda_{1}\right)
\end{aligned}
$$

We have [6]:

$$
E\left[N_{v} \mid N=n\right]=E\left[N_{v}\right]=\frac{\lambda_{0}}{\lambda_{1}}
$$

and

$$
E\left[L_{v} \mid N=n\right]=E\left[L_{v}\right]=\frac{\lambda_{0}}{2 \lambda_{1}^{\frac{3}{2}}}
$$

Define $C_{1}$ to be the total energy used by the sensors in a Voronoi cell to communicate one unit of data to the cluster head. Then, if we approximate the number of hops between a sensor and its $\mathrm{CH}$ at a distance $D$ from it by $D / r$,

$$
E\left[C_{1} \mid N=n\right]=\frac{E\left[L_{v} \mid N=n\right]}{r}
$$

Define $C_{2}$ to be the total energy spent by all the sensors communicating 1 unit of data to their respective cluster heads. Because, there are $n p$ cells, the expected value of $C_{2}$ conditioned on $N$, is given by

$$
\begin{aligned}
E\left[C_{2} \mid N=n\right] & =\sum_{i=1}^{n p} E\left[C_{1} \mid N=n\right] \\
& =n p E\left[C_{1} \mid N=1\right]
\end{aligned}
$$

If the total energy spent by the cluster heads to communicate the aggregated information to the processing center is denoted by $C_{3}$, then

$$
\begin{aligned}
E\left[C_{3} \mid N=n\right] & =\frac{E[D \mid N=n]}{r} \\
& =0,3826 \frac{a^{3}+b^{3}}{a \times b \times r} n p
\end{aligned}
$$

Define $C$ to be the total energy spent in the system. Then:

$$
\begin{aligned}
E[C \mid N=n] & =E\left[C_{2} \mid N=n\right]+E\left[C_{3} \mid N=n\right] \\
& =n p E\left[C_{1} \mid N=1\right]+0,3826 \frac{a^{3}+b^{3}}{a \times b \times r} n p \\
& =n p \frac{E\left[L_{v} \mid N=n\right]}{r}+0,3826 \frac{a^{3}+b^{3}}{a \times b \times r} n p \\
& =n p \frac{\frac{\lambda_{0} \lambda_{1}^{\frac{3}{2}}}{r}}{r}+0,3826 \frac{a^{3}+b^{3}}{a \times b \times r} n p \\
& =n p \frac{\frac{(1-p) \lambda}{2 \sqrt[3]{p \lambda}}}{r}+0,3826 \frac{a^{3}+b^{3}}{a \times b \times r} n p \\
& =\frac{n p}{r} \times \frac{(1-p)}{2 \sqrt{\lambda} \times p^{\frac{3}{2}}}+0,3826 \frac{a^{3}+b^{3}}{a \times b \times r} n p
\end{aligned}
$$

Removing the conditioning on $\mathrm{N}$ yields:

$$
\begin{aligned}
E[C] & =E[E[C \mid N=n] \mid n=N] \\
& =E[N]\left[\frac{p}{r} \times \frac{(1-p)}{2 \sqrt{\lambda} \times p^{\frac{3}{2}}}+0,3826 \frac{a^{3}+b^{3}}{a \times b \times r} p\right] \\
& =\lambda a b\left[\frac{(1-p)}{2 r \sqrt{p \lambda}}+0,3826 \frac{a^{3}+b^{3}}{a \times b \times r} p\right]
\end{aligned}
$$

$E[C]$ minimized by a value of $\mathrm{p}$ that is a solution of

$$
(-1-p) \times a b+c \times \sqrt[3]{p}=0
$$

and is given by

$$
p=\left(\frac{1}{6} \frac{\sqrt[3]{4} \sqrt[3]{a b\left(27 c^{2}+2 a^{2} b^{2}+3 \sqrt{3} \sqrt{27 c^{2}+4 a^{2} b^{2}} c\right)}}{c}(22)\right.
$$




$$
\begin{aligned}
& +\frac{1}{6} \frac{a^{2} b^{2} 4^{2 / 3}}{c \sqrt[3]{a b\left(27 c^{2}+2 a^{2} b^{2}+3 \sqrt{3} \sqrt{27 c^{2}+4 a^{2} b^{2}} c\right)}} \\
& \left.+\frac{1}{3} \frac{a b}{c}\right)^{2}
\end{aligned}
$$

where $c=1,5304 \times\left(a^{3}+b^{3}\right) \sqrt{\lambda}$

The second derivative of the above function is positive; hence, the value of $\mathrm{p}$ given by eq. 23 minimizes the energy spent. The other two roots of eq. 21 are imaginary.

\subsection{Homogeneous Poisson Processes in a Square}

IF $a=b$ then we have the same results like in [3]

\subsection{Homogeneous Poisson Processes in a Circle}

An other important case is when $W_{0}$ is the circle of radius $r_{0}$. Fix the origin and initial line of polar coordinates $r$ and $\theta$ so that the origin is the center of the circle and the initial line is horizontal. We consider the projection of the points $\left(R_{i}, \theta_{i}\right)$, of the Poisson process circularly onto the r-axis $\left(R_{i}\right)$ and radially onto the circumferential $\theta$-axis $\left(\theta_{i}\right)$.

Indeed, consider a two-dimensional homogeneous Poisson process of rate $\lambda$ so that the number $N$ of points in a fixed circular area $C$ of radius $r_{0}$ and area $\pi r_{0}^{2}$ has a Poisson distribution with parameter $\lambda \pi r_{0}^{2}$.

Let $\left(R_{i}, \Theta_{i}\right)$ denote the position of the sensor $s_{i}$ of the process in 2-dimensional space $W_{0}$ (cf Figure 3).

Given the independent random variables $r_{i}$ and $\theta_{i}$ denote the coordinate of the sensor $s_{i}$,

$$
\begin{aligned}
R_{i} & \sim \mathcal{U}\left(\left[0, r_{0}\right]\right) \\
\Theta_{i} & \sim \mathcal{U}([0,2 \pi])
\end{aligned}
$$

with probability densities:

$$
f_{R_{i}}\left(r_{i}\right)= \begin{cases}\frac{2 r_{i}}{r_{0}^{2}} & \text { for } 0 \leq r \leq r_{0} \\ 0 & \text { elsewhere }\end{cases}
$$

and

$$
f_{\Theta_{i}}\left(\theta_{i}\right)= \begin{cases}\frac{1}{2 \pi} & \& \text { for } 0 \leq \theta \leq 2 \pi \\ 0 & \text { elsewhere }\end{cases}
$$

then

$$
\left(R_{i}, \Theta_{i}\right) \sim f_{\left(R_{i}, \Theta_{i}\right)}\left(r_{i}, \theta_{i}\right)
$$

$R_{i}$ and $\Theta_{i}$ are independent and uniformly distributed (iud), then:

$$
f_{\left(R_{i}, \Theta_{i}\right)}\left(r_{i}, \theta_{i}\right)=f_{R_{i}}\left(r_{i}\right) \times f_{\Theta_{i}}\left(\theta_{i}\right)
$$

As per our assumptions, the sensors are distributed according to a homogeneous spatial Poisson process and hence, the number of sensors in a fixed circle area (cf Figure 3 ) is a Poisson random variable, $N$, with mean $\lambda A$, where $A=2 \pi r_{0}^{2}$.

Let us assume that for a particular realization of the process there are $n$ sensors in this area.

Define $D_{i}$ to be the distance between a sensor located at $\left(x_{i}, y_{i}\right), i=1 ; 2 ; \ldots ; n$ and its own cluster head 3 .

$$
\left\{\begin{array}{l}
x_{i}=r_{i} \sin \theta_{i} \\
y_{i}=r_{i} \cos \theta_{i}
\end{array}\right.
$$

Let:

$$
D_{i}=\sqrt{X_{i}^{2}+Y_{i}^{2}}=R_{i}
$$

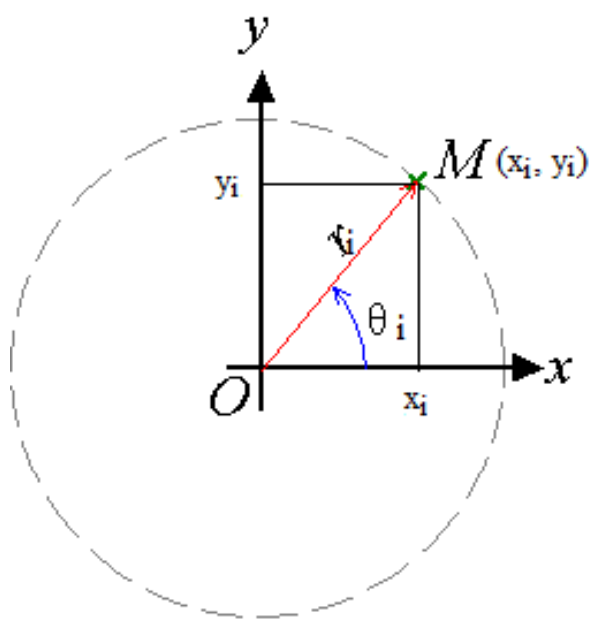

Fig. 3. The radius $R_{i}$

Then the average distance between sensor node and its cluster head is:

$$
\begin{aligned}
E\left[D_{i}\right] & =E\left[R_{i}\right] \\
& =\int_{0}^{2 \pi} \int_{0}^{r_{0}} r_{i} f_{\left(R_{i}, \Theta_{i}\right)}\left(r_{i}, \theta_{i}\right) d r_{i} d \theta_{i} \\
& =\int_{0}^{2 \pi} \int_{0}^{r_{0}} \frac{2 r_{i}^{2}}{2 \pi r_{0}^{2}} d r_{i} d \theta_{i} \\
& =\frac{2}{3} r_{0}
\end{aligned}
$$

There are $n$ sensors uniformly distributed in the circle area,

$$
\begin{aligned}
E[D \mid N=n] & =\sum_{i=1}^{n p} E\left[D_{i}\right] \\
& =n p E\left[R_{i}\right] \\
& =\frac{2}{3} r_{0} n p
\end{aligned}
$$

$C_{3}$ is the total energy spent by the cluster heads to communicate the aggregated information to the base station, then

$$
\begin{aligned}
E\left[C_{3} \mid N=n\right] & =\frac{E[D \mid N=n]}{r} \\
& =\frac{2}{3 r} r_{0} n p
\end{aligned}
$$

$C$ is the total energy spent in the system. Then:

$$
\begin{aligned}
E[C \mid N=n] & =E\left[C_{2} \mid N=n\right]+E\left[C_{3} \mid N=n\right] \\
& =n p E\left[C_{1} \mid N=1\right]+\frac{2}{3 r} r_{0} n p \\
& =n p \frac{E\left[L_{v} \mid N=n\right]}{r}+\frac{2}{3 r} r_{0} n p \\
& =n p \frac{\frac{\lambda_{0}}{2 \lambda_{1}^{\frac{3}{2}}}}{r}+\frac{2}{3 r} r_{0} n p \\
& =n p \frac{\frac{(1-p) \lambda}{2 \sqrt[3]{p \lambda}}}{r}+\frac{2}{3 r} r_{0} n p \\
& =\frac{n p}{r} \times \frac{(1-p)}{2 \sqrt{\lambda} \times p^{\frac{3}{2}}}+\frac{2}{3 r} r_{0} n p
\end{aligned}
$$

Removing the conditioning on $\mathrm{N}$ yields:

$$
E[C]=E[E[C \mid N=n] \mid n=N]
$$




$$
\begin{aligned}
& =E[N]\left[\frac{p}{r} \times \frac{(1-p)}{2 \sqrt{\lambda} \times p^{\frac{3}{2}}}+\frac{2}{3 r} r_{0} p\right] \\
& =\lambda 2 \pi r_{0}^{2}\left[\frac{(1-p)}{2 r \sqrt{p \lambda}}+\frac{2}{3 r} r_{0} p\right]
\end{aligned}
$$

$E[C]$ minimized by a value of $\mathrm{p}$ that is a solution of

$$
3(-1-p)+c \times \sqrt[3]{p}=0
$$

and is given by

$$
\begin{aligned}
p & =-1+\frac{1}{3} c\left(\frac{1}{6} \sqrt[3]{-108+4 \sqrt{-4 c^{3}+729}}\right. \\
& \left.+\frac{2}{3} \frac{c}{\sqrt[3]{-108+4 \sqrt{-4 c^{3}+729}}}\right)
\end{aligned}
$$

where $c=8 r_{0} \sqrt{\lambda}$

The second derivative of the above function is positive; hence, the value of $\mathrm{p}$ given by eq.34 minimizes the energy spent. The other two roots of eq. 32 are imaginary.

\section{CONCLUSION}

To control the energy expenditure of nodes by means of adaptive approach, we have proposed a distributed algorithm for organizing sensors into a hierarchy of clusters with the objective of minimizing the total energy spent in the system to communicate the information gathered by these sensors to an informationprocessing center. Using a realistic environment to check the behavior of this distributed random algorithm and contribute to answer and confirm some results which are approved by theoretical works are very useful to be studied and developed.

\section{REFERENCES}

[1] F. Akyildiz, W. Su, Y. Sankarasubramaniam, and E. Cayirici. A survey on sensor networks. IEEE communications magazine, 40(8):pp. 102-114, 2002.

[2] Adrian Baddeley. Spatial point processes and their applications. Lecture Notes in Mathematics: Stochastic Geometry Springer Verlag , Berlin Heidelberg, 2007.

[3] Seema Bandyopadhyay and E.J. Coyle. An energy efficient hierarchical clustering algorithm for wireless sensor networks. In Twenty-Second Annual Joint Conference of the IEEE Computer and Communications INFOCOM 2003, IEEE Societies, volume vol. 3, pages 1713 - 1723, april 2003.

[4] D. Estrin, L. Girod, G. Pottie, and M. Srivastava. Instrumenting the world with wireless sensor networks. In Proceedings of the International Conference on Acoustics, Speech and Signal Processing, (ICASSP 2001), pages vol. 4,pp. 2033-2036, Salt Lake City, Utah, USA, May 2001.

[5] J.A. Fax and R.M. Murray. Information flow and cooperative control of vehicle formations. Automatic Control, IEEE Transactions on, 49(9):1465-1476, 2004.

[6] S. G. Foss and S. A. Zuyev. On a voronoi aggregative process related to a bivariate poisson process. In Adv. in Appl. Probab, pages 965-981, 1996.

[7] W.R. Heinzelman, A.P. Chandrakasan, and H. Balakrishnan. Energyefficient communication protocol for wireless microsensor networks. In Proceedings of the 33rd Hawaii International Conference on System Sciences (HICSS-33), Hawaii, USA, January 2000.

[8] W.R. Heinzelman, A.P. Chandrakasan, and H. Balakrishnan. An application-specific protocol architecture for wireless microsensor networks. IEEE Transactions on Wireless Communications, 1(4):pp. 660-670, 2002.
[9] P. Holgate. The use of distance methods for the analysis of spatial distributions of points. Stochastic Point Processes, pages pp. 122-35, 1972.

[10] Chalermek Intanagonwiwat, Ramesh Govindan, and Deborah Estrin. Directed diffusion: a scalable and robust communication paradigm for sensor networks. In Proceedings of the 6th annual international conference on Mobile computing and networking, MobiCom '00, pages 56-67, New York, NY, USA, 2000. ACM.

[11] A. Jadbabaie, Jie Lin, and A.S. Morse. Coordination of groups of mobile autonomous agents using nearest neighbor rules. Automatic Control, IEEE Transactions on, 48(6):988-1001, 2003.

[12] S. Kar and J. M F Moura. Distributed average consensus in sensor networks with random link failures. In Acoustics, Speech and Signal Processing, 2007. ICASSP 2007. IEEE International Conference on, volume 2, pages II-1013-II1016, 2007.

[13] S. Karlin. A First Course in Stochastic Processes. Academic Press (USA), 1968.

[14] Nancy A. Lynch. Distributed Algorithms. Morgan Kaufmann Publishers Inc., 1996.

[15] V. Mhatre, C. Rosenberg, D. Kofman, R. Mazumdar, and N. Shroff. Design of surveillance sensor grids with a lifetime constraint. In 1st European Workshop on Wireless Sensor Networks (EWSN), Berlin, January 2004.

[16] Atsuyuki Okabe, Barry Boots, and Kokichi Sugihara. Spatial tessellations: concepts and applications of Voronoi diagrams. John Wiley \& Sons, Inc., New York, NY, USA, 1992.

[17] R. Olfati-Saber, J.A. Fax, and R.M. Murray. Consensus and cooperation in networked multi-agent systems. Proceedings of the IEEE, 95(1):215-233, 2007.

[18] Li Qing, Qingxin Zhu, and Mingwen Wang. Design of a distributed energy-efficient clustering algorithm for heterogeneous wireless sensor networks. Computer Communication, 29(12):2230-2237, 2006

[19] Wei Ren, R.W. Beard, and E.M. Atkins. A survey of consensus problems in multi-agent coordination. In American Control Conference, 2005. Proceedings of the 2005, pages 1859-1864 vol. 3, 2005.

[20] H. R. Thompson. Spatial point processes, with applications to ecology. Biometrika, 42:pp. 102- 115, 1955.

\section{Biographies}

Mostafa SAADI Received the B.Sc. degree in Computer Sciences at the University Hassan $2^{\text {nd }}$, Faculty of Sciences Ain-Chook, Casablanca, Morocco, in 2003, and a M.Sc. degree in Mathematical and Computer engineering at the University Chouaib Doukkali, Faculty of Sciences, El Jadida (FSJ), Morocco, in 2009. He has been working as a professor of Computer Sciences in high school since 2003, in Sidi Rahal Beach, Morocco. Currently, he is working toward his $\mathrm{Ph} . \mathrm{D}$. at FSJ. His current research interests performance evaluation, analysis and simulation of Wireless Sensor networks.

Dr. Moulay Lahcen HASNAOUI Received his Ph.D in modeling and simulation of semiconductor devices at the Paris-Sud University, France (1991-1995). He worked as research associate in developing fuel cell at Department of Engineering Physics, Polytechnic School, Montreal, Canada (1996-1996). He earned his bachelor's degree in Computer Science from University of Montreal, Canada (1998- 2002). Self-employed as a software developer (2002-2004). He worked as research 
assistant professor at Mathematics and Computer Science Department at the Faculty of Sciences, MATIC Laboratory, El Jadida, Morocco, between 2004-2011. He is working as research assistant professor at Computer Sciences Department at the Faculty of Sciences Dhar Al Mahraz, Fez (2011).

Abderrahim BENI HSSANE Is a research and an assistant professor at Science Faculty, Chouab Doukkali University,
El Jadida, Morocco, since September 1994. He got his B.Sc. degree in applied mathematics and his Doctorate of High Study Degree in computer science, respectively, in 1992 and 1997 from Mohamed V University, Rabat, Morocco. His research interests focus on performance evaluation in wireless networks. 